\title{
Hyers-Ulam Stability of Additive Functional Equation Using Direct and Fixed-Point Methods
}

\author{
K. Tamilvanan $\mathbb{D}^{1},{ }^{1}$ G. Balasubramanian, ${ }^{1}$ Nazek Alessa $\left(\mathbb{D},{ }^{2}\right.$ and K. Loganathan ${ }^{3}{ }^{3}$ \\ ${ }^{1}$ Department of Mathematics, Government Arts College for Men, Krishnagiri 635 001, Tamilnadu, India \\ ${ }^{2}$ Department of Mathematical Sciences, Faculty of Science, Princess Nourah Bint Abdulrahman University, Riyadh, Saudi Arabia \\ ${ }^{3}$ Department of Mathematics, Erode Arts \& Science College, Erode 638009, Tamilnadu, India
}

Correspondence should be addressed to K. Loganathan; loganathankaruppusamy304@gmail.com

Received 30 October 2020; Revised 15 November 2020; Accepted 23 November 2020; Published 14 December 2020

Academic Editor: Kottakkaran Sooppy Nisar

Copyright (c) $2020 \mathrm{~K}$. Tamilvanan et al. This is an open access article distributed under the Creative Commons Attribution License, which permits unrestricted use, distribution, and reproduction in any medium, provided the original work is properly cited.

In this present work, we obtain the solution of the generalized additive functional equation and also establish Hyers-Ulam stability results by using alternative fixed point for a generalized additive functional equation $\chi\left(\sum_{g=1}^{l} v_{g}\right)=\sum_{1 \leq g<h<i \leq l} \chi\left(v_{g}+v_{h}+v_{i}\right)-\sum_{1 \leq g<h \leq l} \chi\left(v_{g}+v_{h}\right)-\left(\left(l^{2}-5 l+2\right) / 2\right) \sum_{g=1}^{l}\left(\chi\left(v_{g}\right)-\chi\left(-v_{g}\right) / 2\right)$. where $l$ is a nonnegative integer with $\mathbb{N}-\{0,1,2,3,4\}$ in Banach spaces.

\section{Introduction}

The problem of Ulam-Hyers stability concerns determining circumstances under which, given an approximate solution of a functional equation, one may locate an exact key that is closer to it in some sense. The investigation of stability problem for functional equations is identified to a question of Ulam [1] about the stability of group homomorphisms and affirmatively answered for Banach space by Hyers [2, 3]. It was further generalized and interesting results were obtained by a number of authors [4-6].

Rassias investigated the Hyers-Ulam stability results for the various functional equations in [7-10] through different spaces. Czerwik $[11,12]$ examined the stability of the quadratic functional equation involving several variables in the normed spaces. Numerous mathematicians investigated the various stability results in [13-32].

In 2019, Park et al. [33] introduced additive s-functional inequality. Using the fixed-point method and direct method, he established the Hyers-Ulam stability for the abovementioned one in complex Banach spaces. Also, he examined the Hyers-Ulam stability of homomorphism and derivations in complex Banach algebras. In 2018, Almahalebi
[34] investigated the quadratic functional equation in Banach spaces. And, he established the hyperstability outcome of the same equation through the fixed-point approach.

Radu [35] investigated various results about the stability problem by using the fixed-point alternative. He applied the fixed-point method to examine the stability of Cauchy functional equation and Jensen's functional equations. After his work, numerous authors used the fixed-point method to investigate several functional equations [36-41]. The functional equation

$$
f(x+y)=f(x)+f(y),
$$

is called the Cauchy additive functional equation and it is the most famous functional equation. As $f(x)=c x$ is the solution of (1), every solution of the additive equation is called an additive function.

In this present work, we derive the solution of the generalized additive functional equation along with established Hyers-Ulam stability results by using direct and fixed-point methods for a generalized additive functional equation 


$$
\chi\left(\sum_{g=1}^{l} v_{g}\right)=\sum_{1 \leq g<h<i \leq l} \chi\left(v_{g}+v_{h}+v_{i}\right)-\sum_{1 \leq g<h \leq l} \chi\left(v_{g}+v_{h}\right)-\left(\frac{l^{2}-5 l+2}{2}\right) \sum_{g=1}^{l} \frac{\chi\left(v_{g}\right)-\chi\left(-v_{g}\right)}{2}
$$

where $l \geq 5$ is a nonnegative integer in Banach spaces.

\section{General Solution of the Functional Equation (2)}

In this section, we derive the general solution of the generalized additive functional equation (2).

Here, we consider $\Phi$ and $\Omega$ be real vector spaces.

Theorem 1. If a mapping $\chi: \Phi \longrightarrow \Omega$ satisfies the functional equation (2) for all $v_{1}, v_{2}, \ldots, v_{n} \in \Phi$, then the mapping $\chi: \Phi \longrightarrow \Omega$ satisfies the functional equation (1) for all $x, y \in \Phi$.

Proof. Suppose a mapping $\chi: \Phi \longrightarrow \Omega$ satisfies the functional equation (2). Replacing $\left(v_{1}, v_{2}, v_{3}, \ldots, v_{l}\right)$ by $(0,0,0, \ldots, 0)$ in the functional equation (2), we have $\chi(0)=0$. Replacing $\left(v_{1}, v_{2}, v_{3}, \ldots, v_{l}\right)$ by $(v, 0,0, \ldots, 0)$ in equation (2), we get $\chi(-v)=-\chi(v)$ for all $v \in \Phi$. Therefore, the function $\chi$ is an odd function. Replacing $\left(v_{1}, v_{2}, v_{3}, \ldots, v_{l}\right)$ by $(v, v, 0,0, \ldots, 0)$ in equation (2) and using the property of odd function, we have

$$
\chi(2 v)=2 \chi(v)
$$

$$
\chi\left(2^{2} v\right)=2^{2} \chi(v)
$$

for all $v \in \Phi$. Again, replacing $v$ by $2 v$ in (5) and using (3), we have

$$
\chi\left(2^{3} v\right)=2^{3} \chi(v)
$$

for all $v \in \Phi$. We can generalize for any nonnegative integer $n$ and we get

$$
\chi\left(2^{n} v\right)=2^{n} \chi(v)
$$

for all $v \in \Phi$. Now, replacing $\left(v_{1}, v_{2}, v_{3}, \ldots, v_{l}\right)$ by $(x, y, 0, \ldots, 0)$ in (2), we obtain our desired result of equation (1).

Remark 1. Let $\Omega$ be a linear space and a function $\chi: \Phi \longrightarrow \Omega$ satisfies the functional equation (2). Then, the following claims hold:

(1) $\chi\left(r^{k} v\right)=r^{k} \chi(v)$ for all $v \in \mathbb{R}, r \in \mathbb{Q}, k$ integers

(2) $\chi(v)=v \chi(1)$ for all $v \in \mathbb{R}$ if $\chi$ is continuous

In Sections 3 and 4 , we take $\Phi$ be a normed space and $\Omega$ be a Banach space. For our convincing effortlessness, we describe a function $\Theta: \Phi \longrightarrow \Omega$ as

for all $v \in \Phi$. Replacing $v$ by $2 v$ in (3), we obtain

$$
\begin{aligned}
& \Theta\left(v_{1}, v_{2}, v_{3}, \ldots, v_{l}\right)=\chi\left(\sum_{g=1}^{l} v_{g}\right)-\sum_{1 \leq g<h<i \leq l} \chi\left(v_{g}+v_{h}+v_{i}\right) \\
& -\sum_{1 \leq g<h \leq l} \chi\left(v_{g}+v_{h}\right)-\left(\frac{l^{2}-5 l+2}{2}\right) \sum_{g=1}^{l} \frac{\chi\left(v_{g}\right)-\chi\left(-v_{g}\right)}{2},
\end{aligned}
$$

for every $v_{1}, v_{2}, \ldots, v_{l} \in \Phi$.

\section{Hyers-Ulam Stability of the Functional Equation (2): Direct Method}

In this section, we investigated the Hyers-Ulam stability of the generalized additive functional equation (2) in Banach space by using the direct method.

Theorem 2. Let $\zeta \in\{-1,1\}, \xi: \Phi^{l} \longrightarrow[0, \infty)$ be a mapping such that

$$
\sum_{w=0}^{\infty} \frac{\xi\left(2^{w \zeta} v_{1}, 2^{w \zeta} v_{2}, \ldots, 2^{w \zeta} v_{l}\right)}{2^{w \zeta}}
$$

converges in $\mathbb{R}$ with

$$
\lim _{w \longrightarrow \infty} \frac{\xi\left(2^{w \zeta} v_{1}, 2^{w \zeta} v_{2}, \ldots, 2^{w \zeta} v_{l}\right)}{2^{w \zeta}}=0
$$

for all $v_{1}, v_{2}, \ldots, v_{l} \in \Phi$. If a mapping $\Theta: \Phi \longrightarrow \Omega$ satisfies the inequality

$$
\left\|\Theta\left(v_{1}, v_{2}, \ldots, v_{l}\right)\right\| \leq \xi\left(v_{1}, v_{2}, \ldots, v_{l}\right),
$$

for all $v_{1}, v_{2}, \ldots, v_{l} \in \Phi$, then there exists a unique additive mapping $\Psi: \Phi \longrightarrow \Omega$ satisfying equation (2) and

$$
\|\chi(v)-\Psi(v)\| \leq \frac{1}{2(l-4)} \sum_{w=((1-\zeta) / 2)}^{\infty} \frac{\xi\left(2^{w \zeta} v, 2^{w \zeta} v, 0, \ldots, 0\right)}{2^{w \zeta}},
$$

for all $v \in \Phi$.

Proof. Assume $\zeta=1$. Replacing $\left(v_{1}, v_{2}, \ldots, v_{l}\right)$ by $(v, v, 0, \ldots, 0)$ in $(10)$, we have 


$$
\|(l-4) \chi(2 v)-2(l-4) \chi(v)\| \leq \xi(v, v, 0, \ldots, 0),
$$

for all $v \in \Phi$. From equality (12), we get

$$
\left\|\frac{\chi(2 v)}{2}-\chi(v)\right\| \leq \frac{\xi(v, v, 0, \ldots, 0)}{2(l-4)},
$$

for all $v \in \Phi$. Exchanging $v$ through $2 v$ in (13), we obtain

$$
\left\|\frac{\chi\left(2^{2} v\right)}{2}-\chi(2 v)\right\| \leq \frac{\xi(2 v, 2 v, 0, \ldots, 0)}{2(l-4)}
$$

for all $v \in \Phi$. From (14), we achieve

$$
\left\|\frac{\chi\left(2^{2} v\right)}{2^{2}}-\frac{\chi(2 v)}{2}\right\| \leq \frac{1}{2} \frac{\xi(2 v, 2 v, 0, \ldots, 0)}{2(l-4)},
$$

for all $v \in \Phi$. Adding together (13) and (15), we get the following outcome:

$$
\left\|\frac{\chi\left(2^{2} v\right)}{2^{2}}-\chi(v)\right\| \leq \frac{1}{2(l-4)}\left[\xi(v, v, 0, \ldots, 0)+\frac{\xi(2 v, 2 v, 0, \ldots, 0)}{2}\right],
$$

for all $v \in \Phi$. It follows from (13), (15), and (16), and we can generalize that as follows:

$$
\begin{aligned}
& \left\|\frac{\chi\left(2^{l} v\right)}{2^{l}}-\chi(v)\right\| \leq \frac{1}{2(l-4)} \sum_{w=0}^{l-1} \frac{\xi\left(2^{w} v, 2^{w} v, 0, \ldots, 0\right)}{2^{w}}, \quad \forall v \in \Phi, \\
\Longrightarrow & \left\|\frac{\chi\left(2^{l} v\right)}{2^{l}}-\chi(v)\right\| \leq \frac{1}{2(l-4)} \sum_{w=0}^{\infty} \frac{\xi\left(2^{w} v, 2^{w} v, 0, \ldots, 0\right)}{2^{w}},
\end{aligned}
$$

for all $v \in \Phi$. In order to establish the convergence of the sequence $\left\{\chi\left(2^{w} v\right) / 2^{w}\right\}$, switch $v$ through $2^{s} v$ and also divide by $2^{s}$ in (17). We conclude that, for some $w, s>0$,

$$
\begin{aligned}
\left\|\frac{\chi\left(2^{w+s} v\right)}{2^{(w+s)}}-\frac{\chi\left(2^{s} v\right)}{2^{s}}\right\| & =\frac{1}{2^{s}}\left\|\frac{\chi\left(2^{w+s} v\right)}{2^{w}}-\chi\left(2^{s} v\right)\right\| \\
& \leq \frac{1}{2(l-4)} \sum_{w=0}^{l-1} \frac{\xi\left(2^{w+s} v, 2^{w+s} v, 0, \ldots, 0\right)}{2^{(w+s)}} \\
& \leq \frac{1}{2(l-4)} \sum_{w=0}^{\infty} \frac{\xi\left(2^{w+s} v, 2^{w+s} v, 0, \ldots, 0\right)}{2^{(w+s)}} \longrightarrow 0, \quad \text { as } s \longrightarrow \infty,
\end{aligned}
$$

for all $v \in \Phi$. Therefore, the sequence $\left\{\chi\left(2^{w} v\right) / 2^{w}\right\}$ is a Cauchy. As $\Omega$ is complete, there exists $\Psi: \Phi \longrightarrow \Omega$ so that $\Psi(v)=\lim _{w \longrightarrow \infty}\left(\chi\left(2^{w} v\right) / 2^{w}\right)$ for all $v \in \Phi$. Taking the limit $w \longrightarrow \infty$ in (17), we obtain that result (11) holds for all $v \in \Phi$. To prove that the function $\Psi$ satisfies equation (2), replacing $\left(v_{1}, v_{2}, \ldots, v_{l}\right)$ by $\left(2^{w} v_{1}, 2^{w} v_{2}, \ldots, 2^{w} v_{l}\right)$ and also dividing by $2^{w}$ in (10), we get

$$
\frac{1}{2^{w}}\left\|\Theta\left(2^{w} v_{1}, 2^{w} v_{2}, \ldots, 2^{w} v_{l}\right)\right\| \leq \frac{1}{2^{w}} \xi\left(2^{w} v_{1}, 2^{w} v_{2}, \ldots, 2^{w} v_{l}\right),
$$

for all $v_{1}, v_{2}, \ldots, v_{l} \in \Phi$. Taking the limit $w \longrightarrow \infty$ in the above inequality and using the definition of $\Psi(v)$, we have $\Psi\left(v_{1}, v_{2}, \ldots, v_{l}\right)=0$ for all $v_{1}, v_{2}, \ldots, v_{l} \in \Phi$. Thus, the function $\Psi$ satisfies equation (2). To prove that the function 
$\Psi$ is unique, let $\varphi: \Phi \longrightarrow \Omega$ be another additive mapping satisfying the functional equation (2) and (11). Hence,

$$
\begin{aligned}
\|\Psi(v)-\varphi(v)\| & \leq \frac{1}{2^{s}}\left\|\Psi\left(2^{s} v\right)-\chi\left(2^{s} v\right)\right\|+\left\|\chi\left(2^{s} v\right)-\varphi\left(2^{s} v\right)\right\| \\
& \leq \frac{1}{2(l-4)} \sum_{w=0}^{\infty} \frac{\xi\left(2^{w+s} v, 2^{w+s} v, 0, \ldots, 0\right)}{2^{(w+s)}}, \quad \forall v \in \Phi \\
& \longrightarrow 0 \text { as } s \longrightarrow \infty .
\end{aligned}
$$

Hence, $\Psi$ is unique. Now, replacing $v$ through $(v / 2)$ in (12), we have

$$
\left\|(l-4) \chi(v)-2(l-4) \chi\left(\frac{v}{2}\right)\right\| \leq \xi\left(\frac{v}{2}, \frac{v}{2}, 0, \ldots, 0\right),
$$

for all $v \in \Phi$. The rest of the proof is similar to that when $\zeta=1$. So for $\zeta=-1$, we can prove the results by a similar manner. Hence, the proof is completed.

Corollary 1. Let $\phi$ and $\vartheta$ be positive real numbers. If there exists a mapping $\Theta: \Phi \longrightarrow \Omega$ satisfying the inequality

$$
\left\|\Theta\left(v_{1}, v_{2}, \ldots, v_{l}\right)\right\| \leq\left\{\begin{array}{l}
\phi, \\
\phi\left\{\sum_{i=1}^{l}\left\|v_{i}\right\|^{9}\right\}, \\
\phi\left\{\prod_{i=1}^{l}\left\|v_{i}\right\|^{9}+\sum_{i=1}^{l}\left\|v_{i}\right\|^{l \vartheta}\right\},
\end{array}\right.
$$

for all $v_{1}, v_{2}, \ldots, v_{l} \in \Phi$, then there exists a unique additive mapping $\Psi: \Phi \longrightarrow \Omega$ such that

$$
\|\chi(v)-\Psi(v)\| \leq \begin{cases}\frac{\phi}{|l-4|}, \\ \frac{2 \phi\|v\|^{9}}{(l-4)\left|2-2^{9}\right|} ; & \vartheta \neq 1, \\ \frac{2 \phi\|v\|^{l 9}}{(l-4)\left|2-2^{l 9}\right|} & \vartheta \neq \frac{1}{l},\end{cases}
$$

$$
\begin{aligned}
d(m, n) & =w \Longrightarrow\|m(v)-n(v)\| \leq w \lambda(v) \Longrightarrow\left\|\frac{m\left(\eta_{g} v\right)}{\eta_{g}}-\frac{n\left(\eta_{g} v\right)}{\eta_{g}}\right\| \leq \frac{1}{\eta_{g}} w \lambda\left(\eta_{g} v\right) \\
& \Longrightarrow\|\nu m(v)-\nu n(v)\| \leq \frac{1}{\eta_{g}} w \lambda\left(\eta_{g} v\right) \Longrightarrow d(\nu m(v), \nu n(v)) \leq w L .
\end{aligned}
$$

That is, $d(\nu m, v n) \leq L d(m, n)$. Accordingly, $v$ is a strictly contractive mapping on $\psi$ with Lipschitz constant $L$. From inequality (12), we have for all $v \in \Phi$.

\section{Hyers-Ulam Stability of the Functional Equation (2): Fixed-Point Method}

In this section, we examined the Hyers-Ulam stability of the generalized additive functional equation (2) in Banach space by using the fixed-point method.

Theorem 3. Let $\Psi: \Phi \longrightarrow \Omega$ be a mapping for which there exists a mapping $\xi: \Phi^{l} \longrightarrow[0, \infty)$ and

$$
\lim _{w \longrightarrow \infty} \frac{\xi\left(\eta_{g}^{w} v_{1}, \eta_{g}^{w} v_{2}, \ldots, \eta_{g}^{w} v_{l}\right)}{\eta_{g}^{w}}=0
$$

$\begin{array}{ll}\text { where } \eta_{g} \\ \text { inequality }\end{array}=\left\{\begin{array}{ll}2, & \text { if } g=0, \\ (1 / 2), & \text { if } g=1,\end{array}\right.$ and such that it satisfies the

$$
\left\|\Psi\left(v_{1}, v_{2}, \ldots, v_{l}\right)\right\| \leq \xi\left(v_{1}, v_{2}, \ldots, v_{l}\right),
$$

for all $v_{1}, v_{2}, \ldots, v_{l} \in \Phi$. If there exists a Lipschitz constant $L=L(g)$ such that

$$
v \longrightarrow \lambda(v)=\frac{1}{(l-4)} \xi\left(\frac{v}{2}, \frac{v}{2}, 0, \ldots, 0\right),
$$

has the property

$$
\frac{\lambda\left(\eta_{g} v\right)}{\eta_{g}}=L \lambda(v)
$$

for all $v \in \Phi$. Then, there exists a unique additive mapping $\Psi: \Phi \longrightarrow \Omega$ satisfying equation (2) and

$$
\|\chi(v)-\Psi(v)\| \leq \frac{L^{1-g}}{1-L} \lambda(v),
$$

for all $v \in \Phi$.

Proof. Consider a set $\psi=\{m / m: \Phi \longrightarrow \Omega, m(0)=0\}$ and initiate the generalized metric on $\psi, d(m, n)=$ inf $\{w \in(0, \infty):\|m(v)-n(v)\| \leq w \lambda(v), v \in \Psi\}$. It is easy to view that $(\psi, d)$ is complete. Fix $v: \psi \longrightarrow \psi$ as $v m(v)=$ $\left(1 / \eta_{g}\right) m\left(\eta_{g} v\right)$ for all $v \in \Phi$. For $m, n \in \psi$ and $v \in \Phi$, we have

$$
\|(l-4) \chi(2 v)-2(l-4) \chi(v)\| \leq \xi(v, v, 0, \ldots, 0),
$$

for all $v \in \Phi$. It follows from (30) that 


$$
\left\|\frac{\chi(2 v)}{2}-\chi(v)\right\| \leq \frac{\xi(v, v, 0, \ldots, 0)}{2(l-4)},
$$

for all $v \in \Phi$. Using inequality (27) when $g=0$, we get

$$
\left\|\chi(v)-\frac{\chi(2 v)}{2}\right\| \leq \frac{1}{2} \lambda(v) \Longrightarrow\|\chi(v)-v m(v)\| \leq L \lambda(v),
$$

for all $v \in \Phi$. So we obtain

$$
d(\nu \chi(v), \chi(v)) \leq L=L^{1-g}<\infty,
$$

for all $v \in \Phi$. Replacing $v$ by $(v / 2)$ in (31), we have

$$
\left\|\chi(v)-2 \chi\left(\frac{v}{2}\right)\right\| \leq \frac{\xi((v / 2),(v / 2), 0, \ldots, 0)}{(l-4)},
$$

for all $v \in \Phi$. Using inequality (27) when $g=1$, we have

$$
\left\|2 \chi\left(\frac{v}{2}\right)-\chi(v)\right\| \leq \lambda(v) \Longrightarrow\|v \chi(v)-\chi(v)\| \leq \lambda(v),
$$

for all $v \in \Phi$. Therefore, we obtain the result that

$$
d(\chi(v), v \chi(v)) \leq 1=L^{0}=L^{1-g},
$$

for all $v \in \Phi$. From inequalities (33) and (36), we conclude that

$$
d(\chi(v), v \chi(v)) \leq L^{1-g}<\infty
$$

for all $v \in \Phi$. Next, using fixed-point alternative theorem [35], there exists a fixed point $\Psi$ of $\nu$ in $\psi$ such that

$$
\Psi(v)=\lim _{w \longrightarrow \infty} \frac{\chi\left(\eta_{g}^{w} v\right)}{\eta_{g}^{w}},
$$

for all $v \in \Phi$. In order to establish $\Psi: \Phi \longrightarrow \Omega$ satisfying equation (2), we use an argument similar to that in the proof of Theorem 2. As $\Psi$ is a unique fixed point of $v$ in the set $\Delta=\{((\chi \in \psi) / d(\chi, \Psi))<\infty\}, \Psi$ is a unique function such that

$$
\begin{aligned}
d(\chi, \Psi) & \leq \frac{1}{1-L} d(\chi, \nu \chi) \Longrightarrow d(\chi, \Psi) \leq \frac{L^{1-g}}{1-L} \Longrightarrow\|\chi(v)-\Psi(v)\| \\
& \leq \frac{L^{1-g}}{1-L} \lambda(v),
\end{aligned}
$$

for all $v \in \Phi$. This accomplished the proof.

Corollary 2. Let $\phi$ and $\vartheta$ be positive real numbers. If a mapping $\Theta: \Phi \longrightarrow \Omega$ satisfies inequality (22) for all $v_{1}, v_{2}, \ldots, v_{l} \in \Phi$, then there exists a unique additive mapping such that (23) for all $v \in \Phi$.

Proof. Consider

$$
\xi\left(v_{1}, v_{2}, \ldots, v_{l}\right) \leq\left\{\begin{array}{l}
\phi, \\
\phi\left\{\sum_{i=1}^{l}\left\|v_{i}\right\|^{9}\right\}, \\
\phi\left\{\prod_{i=1}^{l}\left\|v_{i}\right\|^{9}+\sum_{i=1}^{l}\left\|v_{i}\right\|^{l \vartheta}\right\},
\end{array}\right.
$$

for all $v_{1}, v_{2}, \ldots, v_{l} \in \Phi$. Now

$$
\frac{\xi\left(\eta_{g}^{w} v_{1}, \eta_{g}^{w} v_{2}, \ldots, \eta_{g}^{w} v_{l}\right)}{\eta_{g}^{w}}=\left\{\begin{array}{l}
\frac{\phi}{\eta_{g}^{w}} \\
\frac{\phi}{\eta_{g}^{w}}\left\{\sum_{i=1}^{l}\left\|\eta_{g} v_{i}\right\|^{9}\right\}, \\
\frac{\phi}{\eta_{g}^{w}}\left\{\prod_{i=1}^{l}\left\|\eta_{g} v_{i}\right\|^{9}+\sum_{i=1}^{l}\left\|\eta_{g} v_{i}\right\|^{l \vartheta}\right\}, \\
\text { holds. As we have }
\end{array}\right\}\left\{\begin{array}{l}
\longrightarrow 0, \quad \text { as } w \longrightarrow \infty, \\
\longrightarrow 0, \quad \text { as } w \longrightarrow \infty, \\
\text { as } w \longrightarrow \infty .
\end{array}\right.
$$

That is, (24) holds. As we have 
Also,

$$
\lambda(v)=\frac{1}{(l-4)} \xi\left(\frac{v}{2}, \frac{v}{2}, 0, \ldots, 0\right)=\left\{\begin{array}{l}
\frac{\phi}{(l-4)}, \\
\frac{2 \phi\|v\|^{9}}{(l-4) 2^{9}} \\
\frac{2 \phi\|v\|^{l 9}}{(l-4) 2^{l 9}} .
\end{array}\right.
$$

And also,

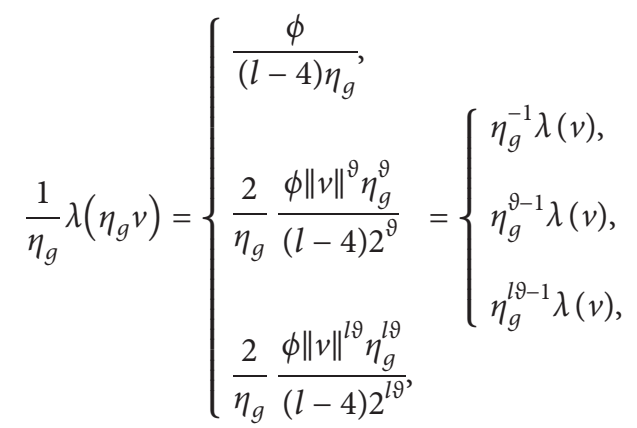

(i) $L=2^{-1}$ if $g=0$ and $L=2^{1}$ if $g=1$

(ii) $L=2^{9-1}$ for $\vartheta<1$ if $g=0$ and $L=2^{1-9}$ for $\vartheta>1$ if $g=1$

(iii) $L=2^{l 9-1}$ for $\vartheta<(1 / l)$ if $g=0$ and $L=2^{1-l 9}$ for $\vartheta>(1 / l)$ if $g=1$

Now, from (28), we verify the following cases:

Case $1: L=2^{-1}$ if $g=0$ :

$\|\chi(v)-\Psi(v)\| \leq \frac{L^{1-g}}{1-L} \lambda(v)=\frac{2^{-1} \phi}{(l-4)\left(1-2^{-1}\right)}=\frac{\phi}{(l-4)}$.

Case 2: $L=2$ if $g=1$ :

$\|\chi(v)-\Psi(v)\| \leq \frac{L^{1-g}}{1-L} \lambda(v)=\frac{\phi}{(l-4)(1-2)}=-\frac{\phi}{(l-4)}$.

Case 3: $L=2^{9-1}$ for $\vartheta<1$ if $g=0$ :

for all $v \in \Phi$. Inequity (2) holds for the following cases:

$$
\|\chi(v)-\Psi(v)\| \leq \frac{L^{1-g}}{1-L} \lambda(v)=\frac{2^{9-1}}{1-2^{9-1}} \frac{2 \phi\|v\|^{9}}{(l-4) 2^{9}}=\frac{2 \phi\|v\|^{9}}{(l-4)\left(2-2^{9}\right)} .
$$

Case 4: $L=2^{1-\vartheta}$ for $\vartheta>1$ if $g=1$ :

$$
\|\chi(v)-\Psi(v)\| \leq \frac{L^{1-g}}{1-L} \lambda(v)=\frac{1}{1-2^{1-9}} \frac{2 \phi\|v\|^{9}}{(l-4) 2^{9}}=\frac{2 \phi\|v\|^{9}}{(l-4)\left(2^{9}-2\right)} .
$$

Case 5: $L=2^{l \vartheta-1}$ for $\vartheta<(1 / l)$ if $g=0$ :

$$
\|\chi(v)-\Psi(v)\| \leq \frac{L^{1-g}}{1-L} \lambda(v)=\frac{2^{l 9-1}}{1-2^{l 9-1}} \frac{2 \phi\|v\|^{l 9}}{(l-4) 2^{l 9}}=\frac{2 \phi\|v\|^{l 9}}{(l-4)\left(2-2^{l 9}\right)} .
$$

Case 6: $L=2^{1-l 9}$ for $9>(1 / l)$ if $g=1$ :

$$
\|\chi(v)-\Psi(v)\| \leq \frac{L^{1-g}}{1-L} \lambda(v)=\frac{1}{1-2^{1-l 9}} \frac{2 \phi\|v\|^{l 9}}{(l-4) 2^{l 9}}=\frac{2 \phi\|v\|^{l 9}}{(l-4)\left(2^{l 9}-2\right)} .
$$


Hence, the proof is accomplished.

\section{Counterexample}

In this section, we examine the following counterexample changed by the well-known counterexample of Gajda [42] to (2).

Example 1. Let a mapping $\Theta: \Phi \longrightarrow \Omega$ be defined by

$$
\Theta(v)=\sum_{l=0}^{\infty} \frac{\phi\left(2^{l} v\right)}{2^{l}},
$$

where

$$
\phi(v)= \begin{cases}\mu v, & -1<v<1, \\ \mu, & \text { otherwise }\end{cases}
$$

where $\mu$ is a constant, and then, the mapping $\Theta: \Phi \longrightarrow \Omega$ satisfies the inequality

$$
\left|\Theta\left(v_{1}, v_{2}, \ldots, v_{l}\right)\right| \leq\left(\frac{l^{2}-5 l+8}{2}\right) 8 \mu\left(\sum_{i=1}^{n}\left|v_{i}\right|\right),
$$

for all $v_{1}, v_{2}, \ldots, v_{l} \in \Phi$, but there exists an additive function $\Psi: \Phi \longrightarrow \Omega$ with a constant $\alpha$ such that

$$
|\chi(v)-\Psi(v)| \leq \alpha|v|
$$

for all $v \in \Phi$.

Proof. It is easy to show that $\chi$ is bounded by $2 \mu$ on $\Phi$. If $\sum_{i=1}^{l}\left|v_{i}\right| \geq(1 / 2)$ or 0 , then the left side of (53) is less than $\left(\left(l^{2}-5 l+8\right) / 2\right) 2 \mu$, and thus, (53) is true. Assume that $0<\sum_{i=1}^{l}\left|v_{i}\right| \geq(1 / 2)$. Then there exists an integer $m$ such that

$$
\frac{1}{2^{(m+2)}} \leq \sum_{i=1}^{l}\left|v_{i}\right|<\frac{1}{2^{(m+1)}} .
$$

So that $2^{m}\left|v_{1}\right|<(1 / 2), 2^{m}\left|v_{2}\right|<(1 / 2), \ldots, 2^{m}\left|v_{l}\right|<(1 / 2)$ and $2^{l} v_{1}, 2^{l} v_{2}, \ldots, 2^{l} v_{l} \in(-1,1)$ for all $l=0,1,2, \ldots, m-1$. So, for $l=0,1, \ldots, m-1$

$$
\begin{aligned}
& \phi\left(\sum_{g=1}^{l} 2^{l} v_{g}\right)-\sum_{1 \leq g<h<i \leq l} \phi\left(2^{l}\left(v_{g}+v_{h}+v_{i}\right)\right)+\sum_{1 \leq g<h \leq l} \phi\left(2^{l}\left(v_{g}+v_{h}\right)\right) \\
& +\left(\frac{l^{2}-5 l+2}{2}\right) \sum_{g=1}^{l} \frac{\phi\left(2^{l}\left(v_{g}\right)\right)+\phi\left(2^{l}\left(-v_{g}\right)\right)}{2}=0 .
\end{aligned}
$$

From the definition of $\chi$, we have

$$
\begin{aligned}
\left|\Theta\left(v_{1}, v_{2}, \ldots, v_{l}\right)\right| & \leq \sum_{i=m}^{\infty} \frac{1}{2^{i}}\left|\phi\left(2^{i} v_{1}, 2^{i} v_{2}, \ldots, 2^{i} v_{l}\right)\right| \\
& \leq \sum_{i=m}^{\infty} \frac{1}{2^{i}}\left(\frac{l^{2}-5 l+8}{2}\right) \mu \\
& \leq\left(\frac{l^{2}-5 l+8}{2}\right) 2^{(1-m)} \mu .
\end{aligned}
$$

It follows from (55) that

$$
\left|\Theta\left(v_{1}, v_{2}, \ldots, v_{l}\right)\right| \leq\left(\frac{l^{2}-5 l+8}{2}\right) 8 \mu\left(\sum_{i=1}^{n}\left|v_{i}\right|\right),
$$

for all $v_{1}, v_{2}, \ldots, v_{l} \in \Phi$. Thus, $\chi$ satisfies (53) for all $v_{1}, v_{2}, \ldots, v_{l} \in \Phi$

Assume on the contrary that there exists a mapping $\Psi: \Phi \longrightarrow \Omega$ additive with a constant $\alpha>0$ satisfying (54). As $\chi$ is bounded and continuous for all $v \in \Phi, \Psi$ is bounded on any open interval containing the origin and continuous at the origin. Hence

By Remark 1, $\Psi$ defined by $\Psi(v)=a v$ for all $v \in \Phi$.

$$
|\chi(v)| \leq(\alpha+|a|)|v|
$$

for all $v \in \Phi$. However, we can select a nonnegative integer $m$ and $m u>\alpha+|a|$. If $v \in\left(0,\left(1 / 2^{m-1}\right)\right)$, then $2^{l} v \in(0,1)$ for all $l=0,1, \ldots, m-1$, and for this $v$, we get

$$
\chi(v)=\sum_{l=0}^{\infty} \frac{\phi\left(2^{l} v\right)}{2^{l}} \geq \sum_{l=0}^{m-1} \frac{\mu\left(2^{l} v\right)}{2^{l}}=m \mu v>(\alpha+|a|) v,
$$

which contradicts.

\section{Conclusion}

In this work, we introduced the generalized finite-dimensional additive functional equation (2) and obtain its general solution in Section 2. In Section 3, we investigated the Hyers-Ulam stability results in Banach space by using the direct method, and in Section 4, we examined the Hyers-Ulam stability results in Banach space by using the fixed-point method. In Section 5, we proved the counterexample changed by the well-known counterexample of Gajda [42] to show the nonstability of the generalized additive functional equation (2).

\section{Data Availability}

No data were used to support the findings of the study. 


\section{Conflicts of Interest}

The authors declare that they have no conflicts of interest.

\section{Acknowledgments}

This research was funded by the Deanship of Scientific Research at Princess Nourah Bint Abdulrahman University through the Fast-track Research Funding Program.

\section{References}

[1] S. M. Ulam, Problems in Modern Mathematics, Wiley, New York, NY, USA, Science Editions, 1964.

[2] D. H. Hyers, "On the stability of the linear functional equation," Proceedings of the National Academy of Sciences, vol. 27, no. 4, pp. 222-224, 1941.

[3] D. H. Hyers, G. Isac, and T. M. Rassias, Stability of Functional Equations in Several Variables, Birkhauser, Basel, Switzerland, 1998.

[4] J. Aczél, Lectures on Functional Equations and Their Applications, p. 509, Academic Press, Cambridge, MA, USA, 1966.

[5] J. Aczél, A Short Course on Functional Equations, Theory and Decision Library. Series B: Mathematical and Statistical Methods, D. Reidel Publishing Co., Dordrecht, Netherlands, 1987.

[6] T. Aoki, "On the stability of the linear transformation in Banach spaces," Journal of the Mathematical Society of Japan, vol. 2, no. 1-2, pp. 64-66, 1950.

[7] J. M. Rassias, "On approximation of approximately linear mappings by linear mappings," Journal of Functional Analysis, vol. 46, no. 1, pp. 126-130, 1982.

[8] J. M. Rassias and H.-M. Kim, "Generalized Hyers-Ulam stability for general additive functional equations in quasi$\beta$-normed spaces," Journal of Mathematical Analysis and Applications, vol. 356, no. 1, pp. 302-309, 2009.

[9] T. M. Rassias, "On the stability of functional equations in Banach spaces," Journal of Mathematical Analysis and Applications, vol. 251, no. 1, pp. 264-284, 2000.

[10] T. M. Rassias, Functional Equations, Inequalities and Applications, Kluwer Acedamic Publishers, Dordrecht, Netherlands, 2003.

[11] S. Czerwik, "On the stability of the quadratic mapping in normed spaces," Abhandlungen aus dem Mathematischen Seminar der Universität Hamburg, vol. 62, no. 1, pp. 59-64, 1992.

[12] S. Czerwik, Functional Equations and Inequalities in Several Variables, p. 420, World Scientific Publishing Co., Inc., River Edge, NJ, USA, 2002.

[13] A. M. Alanazi, G. Muhiuddin, K. Tamilvanan, E. N. Alenze, A. Ebaid, and K. Loganathan, "Fuzzy stability results of finite variable additive functional equation: direct and fixed point methods," Mathematics, vol. 8, no. 7, p. 1050, 2020.

[14] A. L. Cauchy, Analyse Algébrique: Cours d'Analyse de l'École Royale Polytechnique, Éditions Jacques Gabay, Paris, France, 1989.

[15] G. Darboux, "Sur la composition des forces en statique," Bulletin des Sciences Mathématiques et Astronomiques, vol. 9, pp. 281-288, 1875.

[16] P. Gavruta, "A generalization of the Hyers-Ulam-Rassias stability of approximately additive mappings," Journal of Mathematical Analysis and Applications, vol. 184, no. 3, pp. 431-436, 1994.
[17] K.-W. Jun and H.-M. Kim, "On the hyers-ulam stability of a generalized quadratic and additive functional equation," Bulletin of the Korean Mathematical Society, vol. 42, no. 1, pp. 133-148, 2005.

[18] S. M. Jung, Hyers-Ulam-Rassias Stability of Functional Equations in Mathematical Analysis, Hadronic Press, Plan Harbor, FL, USA, 2001.

[19] P. Kannappan, Functional Equations and Inequalities with Applications, Springer Monographs in Mathematics, 2009.

[20] M. Kuczma, An Introduction to the Theory of Functional Equations and Inequalities, Uniwersytet Slaski, Katowice, Poland, 1985.

[21] D. O. Lee, "Hyers-Ulam stability of an additive functional equation," Journal of Applied Mathematics and Computing, vol. 13, no. 1-2, pp. 471-477, 2003.

[22] M. M. Sadr, "On the stability of a generalized additive functional equation," Aequationes mathematicae, vol. 90, pp. 597-606, 2016.

[23] M. S. Moslehian and T. M. Rassias, "Orthogonal stability of additive type equations," Aequationes Mathematicae, vol. 73, no. 3, p. 249, 2007.

[24] C. Park, K. Tamilvanan, G. Balasubramanian, B. Noori, and A. Najati, "On a functional equation that has the quadraticmultiplicative property," Open Mathematics, vol. 18, no. 1, pp. 837-845, 2020.

[25] C. Park, K. Tamilvanan, B. Noori, M. B. Moghimi, and A. Najati, "Fuzzy normed spaces and stability of a generalized quadratic functional equation," AIMS Mathematics, vol. 5, no. 6, p. $7161,2020$.

[26] K. Tamilvanan, J. R. Lee, and C. Park, "Hyers-Ulam stability of a finite variable mixed type quadratic-additive functional equation in quasi-Banach spaces," AIMS Mathematics, vol. 5, no. 6, pp. 5993-6005, 2020.

[27] K. Tamilvanan, J. R. Lee, and C. Park, "Ulam stability of a functional equation deriving from quadratic and additive mappings in random normed spaces," AIMS Mathematics, vol. 6, no. 1, pp. 908-924, 2020.

[28] W. Kavitha, V. Vijayakumar, R. Udhayakumar, and K. S. Nisar, "A new study on existence and uniqueness of nonlocal fractional delay differential systems of order $1<r<2$ in Banach spaces," Numerical Methods for Partial Differential Equations, p. 113. In press, 2020.

[29] K. Kavitha, V. Vijayakumar, R. Udhayakumar, and K. S. Nisar, "Results on the existence of Hilfer fractional neutral evolution equations with infinite delay via measures of noncompactness," Mathematical Methods in the Applied Sciences, p. 118. In press, 2020.

[30] M. Rashid, R. Bibi, A. Kalsoom, D. Baleanu, A. Ghaffar, and K. S. Nisar, "Multidimensional fixed points in generalized distance spaces," Advances in Difference Equations, vol. 2020, no. 1 , p. $571,2020$.

[31] K. S. Nisar, M. Sarfaraz, A. Morsy, and M. K. Ahmad, "Sensitivity analysis for a new class of generalized parametric nonlinear ordered variational inequality problems in ordered Banach spaces," Journal of Inequalities and Applications, vol. 2019, no. 1, p. 249, 2019.

[32] Y. Dong and L. Chen, "On generalized Hyers-Ulam stability of additive mapings on restricted domains of Banach spaces," Aequationes Mathematicae, vol. 90, no. 5, p. 871, 2016.

[33] C. Park, J. R. Lee, and X. Zhang, "Additive s-functional inequality and hom-derivations in Banach algebras," Journal of Fixed Point Theory and Applications, vol. 21, no. 18, p. 14, 2019. 
[34] M. Almahalebi, "Stability of a generalization of Cauchy's and the quadratic functional equations," Journal of Fixed Point Theory and Applications, vol. 20, no. 1, p. 12, 2018.

[35] V. Radu, "The fixed point alternative and the stability of functional equations," Fixed Point Theory, vol. 4, no. 1, pp. 91-96, 2003.

[36] C. Park and Th.M. Rassias, "Fixed points and stability of the Cauchy functional equation," Australian Journal of Mathematical Analysis and Applications, vol. 6, no. 1, pp. 1-9, 2009.

[37] K. Ravi and M. Arunkumar, "On a $n$-dimensional additive functional equation with fixed point alternative," in Proceedings of the ICMS, pp. 314-330, Malaysia, 2007.

[38] W. S. S. Jin, "On the Hyers-Ulam stability of additive quadratic functional equation," International Journal of Inequalities in Pure and Applied Mathematics, vol. 3, no. 3, p. 33, 2002.

[39] I.-i. EL-Fassi, "New stability results for the radical sextic functional equation related to quadratic mappings in (2, $\beta)$-Banach spaces," Journal of Fixed Point Theory and Applications, vol. 20, no. 4, p. 138, 2018.

[40] K. Tamilvanan, G. Balasubramanian, and A. Charles Sagayaraj, "Finite dimensional even-quadratic functional equation and its Ulam-Hyers stability," AIP Conference Proceedings, vol. 2261, p. 030002, 2020.

[41] K. Tamilvanan, A. Nandhakumar, and P. Muthukumar, "Solution and stability of finite dimensional quadratic functional equation in Banach spaces," AIP Conference Proceedings, vol. 2261, p. 030057, 2020.

[42] Z. Gajda, "On stability of additive mappings," International Journal of Mathematics and Mathematical Sciences, vol. 14, Article ID 817959, 4 pages, 1991. 